\title{
Idiosyncratic Behavior of Globally Distributed Manufacturing
}

\author{
Stanislaw Strzelczak \\ Warsaw University of Technology, Faculty of Production Engineering, Warsaw, Poland \\ s.strzelczak@wip.pw.edu.pl
}

\begin{abstract}
The paper presents results of empirical research, which explores systemic background of increasing turbulences and disruptions within globally distributed manufacturing networks. Among the identified factors three have biggest impact: (1) the level of completeness and connectivity of the networks, i.e. topological characteristics of the manufacturing network (2) the herd behavior of clients and decision makers, which enhances or tames the demand due to occasional asymmetry of their perception of the demand (3) the diversity of operational environments within the network, which itself may be a dominant factor of turbulences or even disruptions of the operational processes. It means that in some circumstances, the internal resources of companies may have limited value as a countermeasure against the unlikely effects of turbulences and disruptions. The research has also identified some other factors of idiosyncratic behavior of globally distributed manufacturing, which are rooted in some particular operational policies.
\end{abstract}

\section{Introduction}

The industrial practice of recent years brings more and more examples of idiosyncratic behavior of globally distributed manufacturing networks [6, 12]. In some circumstances industrial supply chains present surprisingly sensitive reactions to the disruptive events or even minor changes in the business environment, like reversing trend of demand. Disruptions and turbulences are becoming more and more frequent, like the catastrophic events, which often trigger them. In extreme cases we can observe long lasting breakdowns of business continuity or even bankruptcy avalanches. The existing theories, which are mostly rooted in the industrial dynamics [14], the bullwhip effect related research [1] and enterprise risk management [11], when confronted with the empirical evidence, provide only limited explanation to the increasingly frequent phenomena, as a limited scope of probably factors of idiosyncratic behavior is usually considered in the literature [15].

The purpose of the research presented in this paper was to investigate the reasons and mechanisms, which influence emergence and dynamics of idiosyncratic phenomena in manufacturing networks, with a special reference to the circumstances and qualities, which are typical for globally distributed manufacturing networks, like cross-border effects, increased and more variable lead times, or diversified business environments. 
To avoid disadvantages of the earlier research, presented in the available literature, a holistic methodological approach was presumed from the early beginning, including hybrid and multi-perspective modeling. A particular attention was given to the explanation of complexity of all existing reasons of idiosyncratic behavior, by empirical evidence. This was protected by a range of research approaches, ranging from case studies and interviewing, through analysis of operational records from the ERP databases, to a hybrid modeling.

\section{Existing Knowledge}

The existing theory presents two major explanations of unsteady behavior of industrial networks, both linked to demand information distortion backward supply chains:

1. Misperception of feedback in the closed loops of decision making, typically enhanced by some operational factors, like lot-sizing, etc. [13],

2. Behavioral causes, e.g. shortage gaming by decision makers [5].

The theory suggests, that the most steady demand patterns can be observed at the end of supply chains, while the final customers are not considered as a major source of disruptions and operational risks. It also means that it is taken for granted, that the distortion of demand information normally increases backward supply chain. Typically the available publications discuss behavioral effects without any consideration of the social context or the social dynamics. Particularly, the impacts of communication within industrial networks or other impacts of external environment, like the media, which all together may possibly cause misperceptions, herd behaviors and other behavioral impacts resulting in deviation of decision making from its normal mode, are not being considered in the production management literature. It is also worth to notice, that the conclusions from the research around prospect theory were not considered by the production management literature [2].

In recent several years numerous publications exploited different frameworks of operational risk management. The factors of risk are being classified in different ways and risk prevention and mitigation policies proposed [e.g. 4, 3, 9, 10, 16], together with some relevant performance measurements [8]. It is notable, that this stream of literature actually applies a particular research perspective. The explored phenomena are being considered as affecting a single company, and locally applied countermeasures are the focus of considerations. This happens, despite the considered factors are located not only inside a company, but also outside, in the supply chain. Furthermore, some external determinants, like oscillating or even disrupting exchange rates, or surprisingly - the effects of global spread or off-shoring of production processes (e.g. longer and more variable lead times), got very limited attention in the literature as a factor of turbulences within industrial networks.

To resume, due to a reductionism of the research methodologies in the literature, a question is justified, if some important factors were ignored, e.g. some exogenous determinants or structural qualities or social behaviors or systemic factors. The above doubts established the starting point of the presented research. 


\section{$3 \quad$ Methodology and Data}

The formulated problem demanded for a research, which could identify all actual reasons of idiosyncratic behavior of globally distributed manufacturing networks. Hence, a holistic model approach was needed and an access to a relevant data. Employing a causal texture framework for organizational environments [7], the below ontological model of problem domain has been developed, which eliminate the methodological shortcomings of former research (Fig.1). It recognizes environmental heterogeneity, different types of determinants and different types of interdependencies, which may trigger or enhance idiosyncratic phenomena.

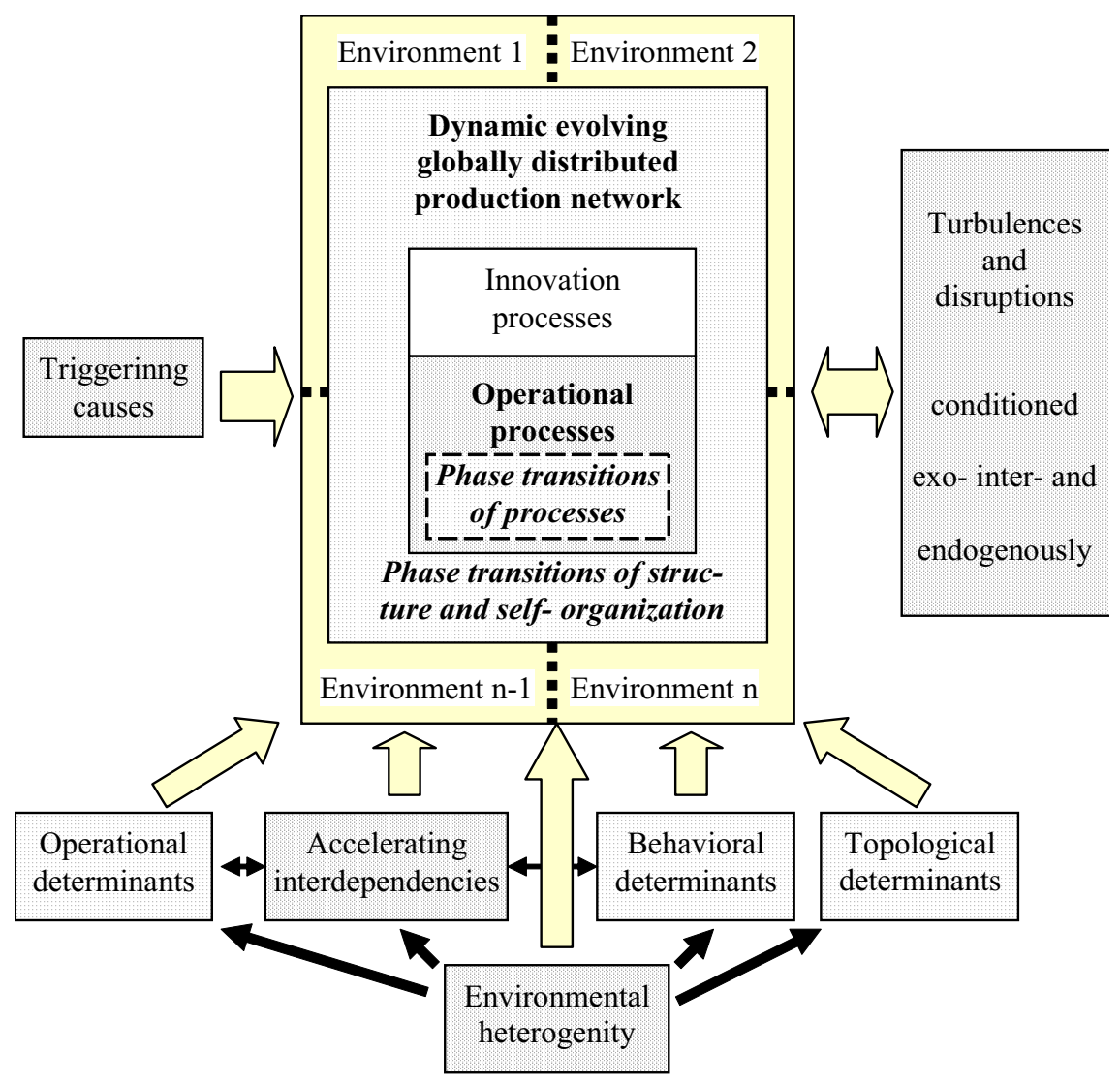

Fig. 1. Overall model of the problem domain

After consideration of possibilities to access relevant data, three approaches for empirical data collection were assumed, to protect reliability and relevance of final results of the research, and particularly to support later modeling and simulations: 
- field observation or case studies, including case studies of turbulent phenomena within network context, like disruption avalanches;

- semi-structured interviewing and questionnaire research;

- exploration of databases of the ERP systems of networked companies, as well as other available statistical data.

Table 1. Structure of the research

\begin{tabular}{|l|l|l|}
\hline \multicolumn{1}{|c|}{ Phase of research } & \multicolumn{1}{|c|}{ Scope of research } & \multicolumn{1}{c|}{ Methods and tools } \\
\hline Initial (conceptual) & $\begin{array}{l}\text { Initial problem conceptualization } \\
\text { Identification of factors and ele- } \\
\text { ments of the problem domain }\end{array}$ & $\begin{array}{l}\text { Analysis of literature } \\
\text { Questionnaire research } \\
\text { Semi-structured interviews }\end{array}$ \\
\hline Ontological & $\begin{array}{l}\text { Conceptualization of domain } \\
\text { Identification of interdependencies } \\
\text { Assumptions for hybrid modeling }\end{array}$ & $\begin{array}{l}\text { Database and statistics analysis } \\
\text { Semi-structured interviews } \\
\text { Influence diagrams }\end{array}$ \\
\hline $\begin{array}{l}\text { Experimental and } \\
\text { monographic research }\end{array}$ & $\begin{array}{l}\text { Development of hybrid models } \\
\text { Calibration of models } \\
\text { Simulations }\end{array}$ & $\begin{array}{l}\text { Bayesian-Belief Networks } \\
\text { Management games } \\
\text { Hybrid simulations }\end{array}$ \\
\hline $\begin{array}{l}\text { Analysis and synthesis } \\
\text { of results }\end{array}$ & $\begin{array}{l}\text { Results assessment } \\
\text { Identification of phase transitions }\end{array}$ & $\begin{array}{l}\text { Statistical and spectral analysis } \\
\text { Expert panels } \\
\text { Scenario modeling }\end{array}$ \\
\hline
\end{tabular}

The further research was run according to a plan, which is presented with more details in the above table. The empirical data were collected during several research and advisory projects, leaded by the main author. The data were collected typically in supply chains, not single companies, to get the possibility for empirical analysis of systemic driven phenomena. The data were collected in companies from five European countries (UK, Germany, Poland, Czech Republic and Slovakia) and China.

The analysis of cause and effects relations was supported by cognitive premapping and development of influence diagrams. Descriptive measurement scales were developed to enable hybrid assessments of leverages between behavioral and material phenomena. A particular approach was developed to enable researching of impacts of some social phenomena. E.g. an analysis of social modes accompanying herd behaviors, which can be reflected in a social communication or by some decision making related behaviors, can be run this way. We can do it by confrontation of frequencies of some phrases in the social communication (e.g. at some web-sites or forums etc.) with the changes of decision making patterns, e.g. reflected by under- or over-ordering. It means that social modes can be measured and monitored, including follow-up of social media by Internet. A statistical analysis of the research results (Pearson - for correlations and Cronbach's Alpha - for reliability) was also applied.

The hybrid modeling was based upon agency theory. However, use of AnyLogic package, enabled to incorporate behaviors and social interactions of agents. Because of that modeling of social dynamics within the production networks and between the agents and environment became possible. E.g. it became possible to model increasing fears or euphoria, leading to herd behavior of decision makers. Different operational strategies and policies could be also considered this way, e.g. comparison of lean 
manufacturing networks and networks with high level of connectivity, like the cluster-based networks, or comparison of push- and pull-flow based control, etc.

\section{$4 \quad$ Results of the Research}

The first two stages of the research confirmed, that idiosyncratic behavior of globally distributed manufacturing networks is a systemic phenomena driven by inter- endoand exogenous interdependencies, and determined by following factors:

- triggering causes:

- turbulences in the environment,

- disruptions, which immediately influence operation of network etc.,

- deviations of processes or some operational characteristics,

- enabling causes:

- accelerating interdependencies: exo-, inter- and endogenous,

- heterogenic environment (or its segments), process flow barriers between them,

- conditioning causes:

- network topology:

○ topology of flows,

o topology of technical and organizational tiers,

- behavioral determinants:

o profile of inter-organizational behaviors,

o profile of asymmetric perception of demand during purchasing decisions,

- operational determinants:

○ profile of operational liquidity,

o profile of operational policies,

o profile of operational risks.

The research provided empirical evidence for a thesis, that among the different nonbehavioral factors three have the biggest impact on idiosyncratic phenomena:

- the level of completeness and connectivity of the networks, which is related to the freedom of choosing alternative vendors or distribution channels,

- the diversity of operational environments within the network, which is related to diversification of operational characteristics of different segments of the network,

- the profile of operational liquidity ${ }^{1}$, which is related to ability to cope with changing market demand and availability of supplies, that is depending not only to the internal resources and contingencies, but also to the internal operational flexibility.

The research has also shown some other factors of turbulent behavior of manufacturing networks, which are rooted in particular operational policies ${ }^{2}$. Oppositely, it has shown that common contingencies, like inventories, may have limited role as countermeasures against disruptions or turbulences, depending on some circumstances.

\footnotetext{
${ }^{1}$ To be distinguished from financial liquidity.

${ }^{2}$ Not discussed in this paper due to its limited size.
} 


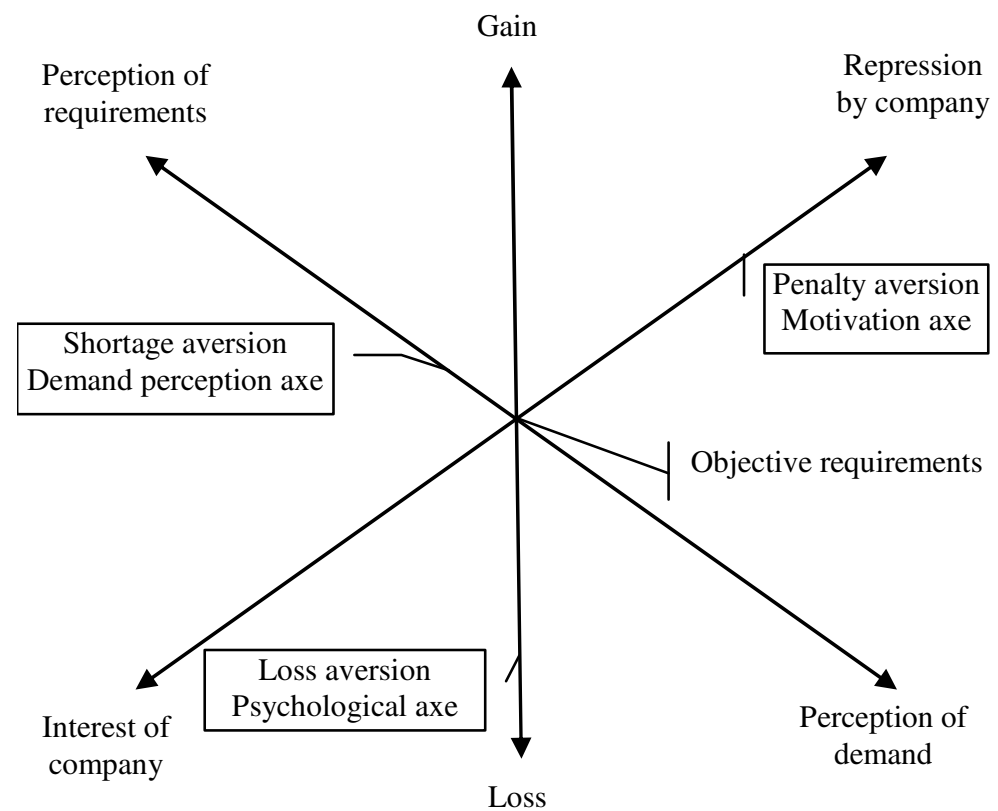

Fig. 2. Asymmetric perceptions behind operational purchasing decisions

The research of behavioral causes of idiosyncratic phenomena in manufacturing networks suggest, that a particular mechanism is underlying misperception of demand and later its distortion, which is different than that described in the literature of bullwhip effect and by the theory of prospect. It is presented at Figure 2. Three asymmetries work together which may deviate perception of purchasing requirements from the actual objective level: fear of loss by a company, fear of a material shortage and fear personal loss due to some penalty from a company, depending on the motivation system. The fear of material shortage (or surplus) can be substantially enhanced by communication with the environment: clients and vendors or the overall environment, particularly markets. Occasionally a herd behavior of clients and/or decision makers may arise, like panic or euphoria, which may enhance or tame the demand or supplies. If the speed of communication is high, like it happens with the Internet based media, and if the change of supplies or demand is not limited by availability of some resources, the turbulence within the network maybe extremely rapid, high and long lasting. Its growth is driven by an accelerating interdependence of modes at markets and decision-making behaviors. Due to increasing openness of social communication this phenomena may have a growing impact on manufacturing industries and economies in the near future. To continue, it is interesting, that the research has confirmed, that by a comparison of operational records from the ERP databases and the records of media or business communication (e.g. content of particular websites or forums, etc.), we can follow and confirm correlation of changing asymmetries of the demand perceptions and changing modes of markets or other communities (like the communities of decision makers within one network). 
The results of both, case studies and hybrid modeling have confirmed that disruptions and turbulences propagate within networks, like avalanches in different directions, depending on the triggering causes and environmental factors. This means that the explanations of bullwhip effect by existing theories cannot be recognized as a relevant framework for a full explanation of the discussed phenomena.

Although the results presented in this paper were obtained on the basis of research and advisory projects, done in industrial companies, which operate in the EU and China, they obviously apply to other sectors regions. Nevertheless, the proposed methodology and the research results should be considered as initial. They need to be verified and developed by a further empirical and conceptual research.

\section{Conclusions and Summary}

The obtained results have extended available knowledge on the reasons and mechanisms of disruptions and turbulences within globally distributed manufacturing networks. Systemic factors of disruptions and turbulences have been identified and the model of their impact has been proposed and justified. Among the identified factors two may have a growing impact in the future: the herd behavior of decision makers driven by growing possibilities of social communication and the diversity of network environments due to the increasing globalization.

The obtained results put a question mark against common operational strategies, particularly these, which are rooted in the concept of lean manufacturing. Lean networks are typically characterized by a minimized number of clients and vendors as well as by limited contingencies of resources, like capacities or inventories. Using the results of the research we can argue that lean networks are particularly exposed to disruptions and turbulences and by leaning the network we increase its potential for idiosyncratic behaviors. Similarly, according to the obtained results, the global spread of manufacturing networks, due to increased diversity of operational environments and due to deteriorated operational characteristics, is normally enhancing idiosyncratic performance of manufacturing networks, which up to now was not considered within operation strategy processes. Particularly, the operational strategies and policies should consider differences of operational environments along the supply chains, mostly these, which influence operational liquidity and connectivity. There are good reasons to advocate for further research aimed at methodologies and assessments which could facilitate these shortcomings.

Referring to the possible practical implementations of the obtained results, two possible mainstreams seem to have major role: executive education and consultancy. Using the existing experience it is possible to propose and justify some frameworks means, which can be used to improve strategic planning of operations and managing the performance of globally distributed production networks, by harmonization of strategies, systems and cultures. However, a major difficulty cannot be easily avoided. The companies tend to perceive reality from the perspective of their interior, while the discussed phenomena has evidently systemic roots and should be considered from a network perspective, including interactions with the business environment. 
To resume, it is worth to note some novelty of the research and obtained results. The material and cultural phenomena and processes within industrial networks were considered simultaneously. This enabled new modeling approaches, and much more relevant explanations of the discussed phenomena were obtained, than before. The results suggest new types of threats and perils, which may follow lean networking, global outsourcing and off-shoring. Finally, a new way of parallel researching of social phenomena observed in media and operational processes observed in the operational databases was proposed.

\section{References}

1. Bhattacharya, R., Bandyopadhyay, S.: A review of the causes of bullwhip effect in a supply chain. The International Journal of Advanced Manufacturing Technology 54(9-12), 1245-1261 (2010)

2. Camerer, C., Loewenstein, G., Rabin, M. (eds.): Advances in behavioral economics, pp. 689-723. Princeton University Press, Princeton (2004)

3. Chopra, S., Sodhi, M.S.: Managing risk to avoid supply-chain breakdown. Sloan Management Review 46(1), 53-62 (2004)

4. Christopher, M., Holweg, M.: "Supply Chain 2.0": managing supply chains in the era of turbulence. International Journal of Physical Distribution and Logistics Management 41(1), 63-82 (2011)

5. Croson, R., Donohue, K.: Behavioral causes of the bullwhip effect and the observed value of inventory information. Management Science 52(3), 323-336 (2006)

6. Dowell, A.: Effects of Triple Disaster Will Reverberate for Months. The Wall Street Journal (March 28, 2011)

7. Emery, F.E., Trist, E.L.: The causal texture of organizational environments. Human Relations 18(1), 21-32 (1965)

8. Fransoo, J., Wouters, M.: Measuring the bullwhip effect in the supply chain. Supply Chain Management: An International Journal, 5 5(2), 78-89 (2000)

9. Jüttner, U.: Supply chain risk management. Understanding the business requirements from a practitioner perspective. International Journal of Logistics Management 16(1), 120-141 (2005)

10. Kleindorfer, P.R., Saad, G.H.: Managing disruption risks in supply chains. Production and Operations Management 14(1), 53-68 (2006)

11. Pfohl, H.-C., Köhler, H., Thomas, D.: State of the art in supply chain risk management research: empirical and conceptual findings and a roadmap for the implementation in practice. Logistics Research 2(1), 33-44 (2010)

12. Schäfer, D.: Lack of parts hits VW production. Financial Times (January 20, 2011)

13. Sterman, J.D.: Misperceptions of feedback in dynamic decision making. Organizational Behavior and Human Decision Processes 43(3), 301-335 (1989)

14. Sterman, J.D.: Business dynamics. McGraw-Hill (2000)

15. Strzelczak, S.: Reducing turbulences in industrial supply chains. In: Koch, T. (ed.) Learn Business System and Beyond. LNBIP, vol. 257, pp. 393-402. Springer, Boston (2008)

16. Wagner, S.M., Bode, C.: An empirical investigation of supply chain performance along several dimensions of risk. Journal of Business Logistics 29(1), 307-325 (2008) 